\title{
Comparative Study of Enhanced AODV Routing Protocols in VANET
}

\author{
Amit Joshi \\ M.Tech Scholar \\ Graphic Era Hill University \\ Dehradun, Uttarakhand-India
}

\author{
Priyanka Sirola \\ M.Tech Scholar \\ Graphic Era Hill University \\ Dehradun, Uttarakhand-India
}

\author{
Kamlesh C. Purohit \\ Assistant Professor \\ Graphic Era University \\ Dehradun, Uttarakhand-India
}

\begin{abstract}
Vehicular Ad-hoc network (VANET) is a self-organizing and infrastructure less network i.e. used to provide communication between Vehicle-to-Vehicle and Vehicle to Road Side Units (RSUs). It is one of the influencing research area that have been used in wireless communication for the better improvement of Intelligent Transportation Systems (ITS) because it has tremendous potential to provide a safe and comfortable environment for the driver and passenger along with allied services in the cases of emergencies. The primary purpose of VANET is to support vehicular safety, passenger security, traffic monitoring and other commercial application. Currently, a lot of research work has been carried out on several specific areas like Routing, Security and Quality of Service (QoS). However, VANET has very dynamic topology in nature, in large \& variable network size and constrained mobility; these characteristics led to the need for efficient routing and resource saving protocol. The most widely used protocol in VANET is AODV. There are several enhancements which are proposed in AODV. In this paper, we have studied \& analyzed several AODV enhancements as well as their comparative analysis in a realistic VANET context.
\end{abstract}

\section{General Terms}

Standardization, Design, Performance

\section{Keywords}

Vehicular Ad-hoc Network (VANET), Intelligent Transportation System (ITS), AODV, NS-2

\section{INTRODUCTION}

As we all know, thousands of people across the world died every year in car accidents, therefore in most of the countries various safety information like traffic lights \& speed limits are used, but still it is not a beneficial solution. Many government and major automotive industries accepted that vehicular safety is going to be a big challenge [1].

So as a result, to improve traffic safety along with the lifestyle of the people a new advanced technology is emerged i.e. Vehicular Ad-hoc Network (VANET). It's an advance form of wireless communication technology [2]. Apart from vehicular safety and security, there are lots of features that can improve passenger comfort like web browsing, information updates etc... Figure 1 shows the different components of the generalized architecture used in VANET. VANET is an advance technology in which vehicles are used as mobile nodes to create a robust wireless ad-hoc network. This type of ad-hoc network builds up in between Vehicle-to-Vehicle and Vehicleto-RSUs (Road Side Units).

Vehicular Ad-hoc Network (VANET) is an extend form of Mobile Ad-hoc Network (MANET), but moving nodes are vehicles in it.

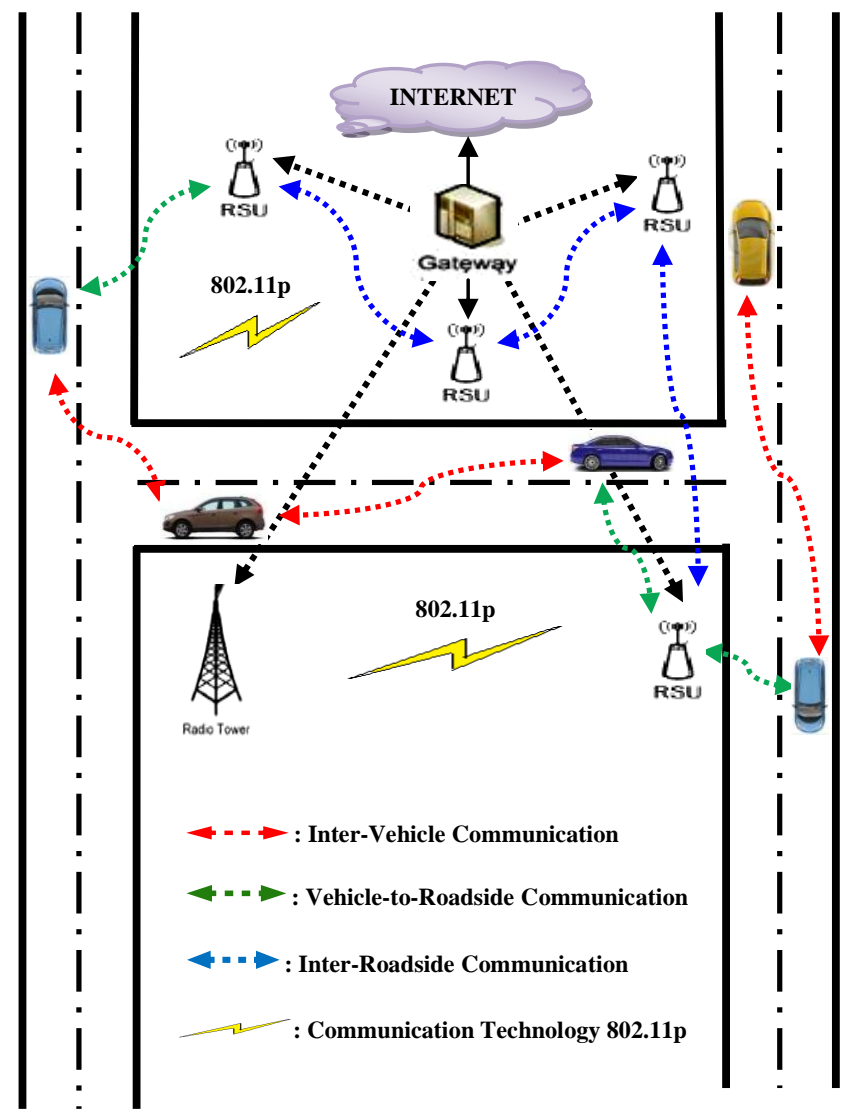

Figure 1: Generalized Architecture in VANET

Moving nodes in VANET performs two types of functions; as a participant in Inter-Vehicular communication and act as a router in the network for data forwarding and routing between intermediate nodes that lies in the transmission range of that node [3].

There are two types of nodes which are used in VANET i.e. static nodes and mobile nodes. Mobile nodes are also called as On-board Units (OBUs). OBUs are computer controlled devices which are mounted on each and every vehicle in the network. Static nodes are Road-Side Units (RSUs), which are located at intersections, near the road and parking lots. An efficient benefit of RSU is that it acts as a gateway in many VANET applications such as internet surfing \& map download etc. 

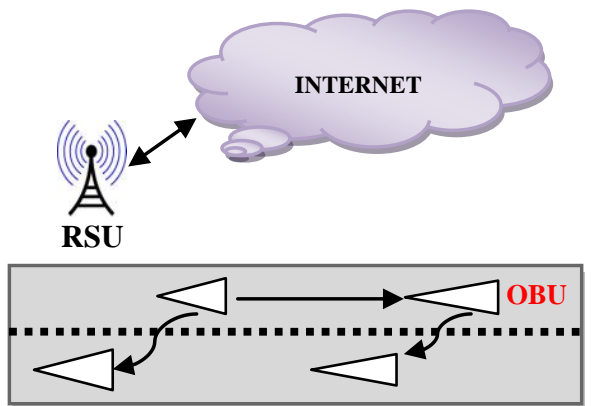

Figure 2: Node types in VANET

To establish a VANET, IEEE has defined the standard 802.11p. The main objective of this is to describe the approach introduce by the IEEE 802.11p WAVE. WAVE stands for "Wireless Access in Vehicular Environment". WAVE makes changes in the overall IEEE 802.11 standard. The allocation of the Dedicated Short Range Communication (DSRC) spectrum arise this standardization process which is allocated by U.S. Federal Communications Commission (FCC) in 1999. This Commission allocated $75 \mathrm{MHz}$ of spectrum in the $5.9 \mathrm{GHz}$ band for DSRC to be exclusively used for V2V \& V2I communication. In contrast to regional standards of DSRC, WAVE is the only standard which can be followed across the world. The functionality of these standards is to define how applications work in the WAVE environment. Table 1 shows protocols, their standards and the corresponding layers in the OSI model [4] [5].

Though VANET have some characteristics like self organized infrastructure less network, which are common with wireless ad-hoc network, VANET also have some unique characteristics that makes it efficient and robust. Some of these characteristics are as follows [6] [7]:

i. High mobility of nodes.

ii. Rapidly changing network topology.

iii. Unbounded network size.

iv. Real-time, time-sensitive data exchange.

v. Crucial effect of security \& privacy.

vi. Adequate energy \& storage capacity.
As per the DSRC, there are lots of VANET applications. These applications can divide into two main categories i.e. Safety and Non-safety applications. We can further classify these applications such as Safety (time \& life critical), Traffic Management, Improve Driver Comfort and Maintenance. Figure 3 shows a generalized view of VANET applications [8] [9].

As per the literature survey, we find that the routing protocols in VANET are originated from MANET routing protocols like AODV, DSR etc...

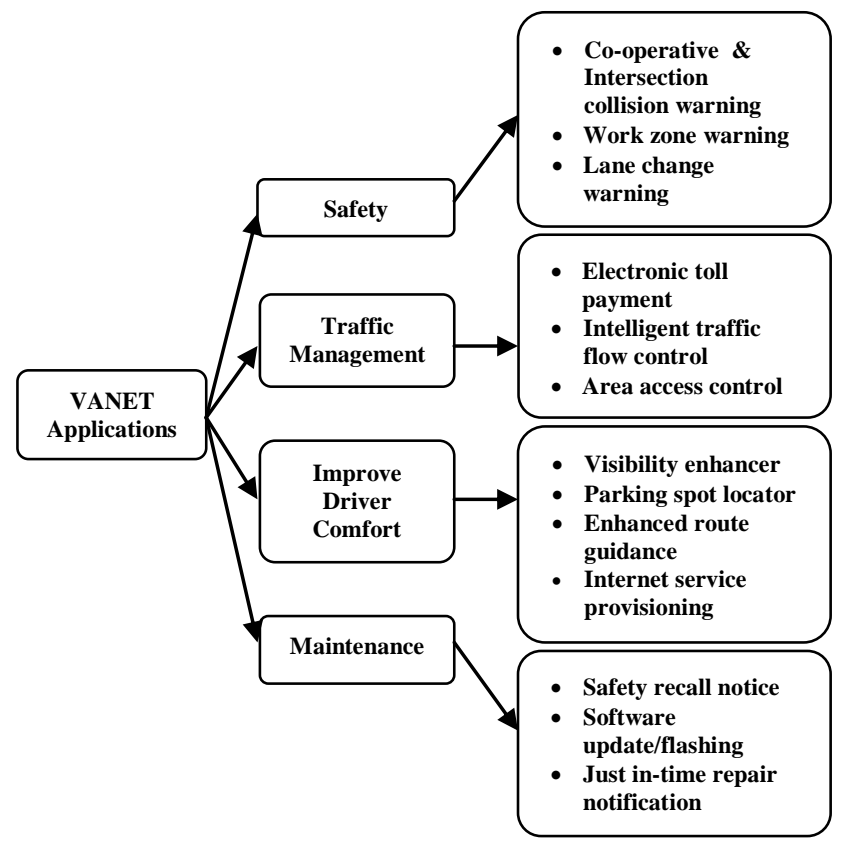

Figure 3: VANET Applications

Because of VANETs unique characteristics like highly dynamic network, lots of research work have been performed for the development of routing protocols. The key consideration for designing a routing protocol is to provide a best possible path between vehicles by minimizing the end-to-end delay, overhead and other important parameters.

Table 1: A list of protocols in WAVE communication Stack

\begin{tabular}{|l|l|l|l|}
\hline \multicolumn{1}{|c|}{ PROTOCOLS } & STANDARDS & \multicolumn{1}{|c|}{ PURPOSE OF THE STANDARD } & $\begin{array}{c}\text { OSI MODEL } \\
\text { LAYER }\end{array}$ \\
\hline WAVE resource manager & IEEE 1609.1 & $\begin{array}{l}\text { It describes an application by which OBUs communicate with } \\
\text { computational resources \& other complex processes which are } \\
\text { operated outside the OBUs. }\end{array}$ & Not Applicable \\
\hline WAVE security services & IEEE 1609.2 & $\begin{array}{l}\text { It provides us security services for better communication \& to } \\
\text { secure our messages. }\end{array}$ & Not Applicable \\
\hline WAVE networking services & IEEE 1609.3 & It provides routing \& forwarding services within a system. & $2,3 \& 4$ \\
\hline Multichannel Operation & IEEE 1602.4 & $\begin{array}{l}\text { Enhanced the IEEE 802.11p MAC to support multichannel } \\
\text { operation. }\end{array}$ & 2 \\
\hline WAVE PHY \& MAC & IEEE 802.11p & $\begin{array}{l}\text { It indicates the functions of MAC \& PHY layers which are } \\
\text { required to work in a dynamic vehicular environment i.e. } \\
\text { VANET. }\end{array}$ & $1 \& 2$ \\
\hline
\end{tabular}


Because of the hierarchical principle of the MANET protocols (AODV, DSDV), there is a problem of designing a protocol. Highly dynamic nature of VANET make variable link for data transmission. Thus, these common routing protocols which are used in MANET can't satisfy the performance requirements of VANET. Lots of protocols have been proposed in recent years. Out of these proposed protocols, Ad-hoc On Demand Distance Vector (AODV) routing protocol is most significant \& commonly used protocol. It is a reactive, single path, on- demand routing protocol. As we mention earlier that hierarchical design of AODV protocol can't satisfy the performance of VANET. So in this paper we present and analyze various AODV enhancements which occur over and over years as well as their comparative study in a realistic VANET context [10].

The remainder of this paper is organized as follows: Section 2 introduces a brief overview of AODV protocol. In section 3 we present several AODV enhancements in context to realistic VANET. Relative comparison of all the enhancements is describes in section 4. Finally, section 5 presents our conclusion and gives the future prospective about the current research in this area.

\section{AD-HOC ON DEMAND DISTANCE VECTOR (AODV) PROTOCOL}

AODV is one of the most widely used on-demand reactive routing protocol. Perkins et al. [11] who created this protocol gives a detail study and analysis of this protocol for the adhoc networks. It's a common reactive protocol which is used in ad-hoc networks. It's a single path routing protocols and it consist of two main phases; the first phase in which route is discovered i.e. Route Discovery phase and the second phase is Route Maintenance phase in which broken links are repaired. The Route Discovery phase uses two cycles i.e. Route REQest (RREQ) \& Route REPly (RREP). Route Discovery phase is initiated whenever a source node wishes to sends a packet or a message to a destination node.

Source node transmits RREQ to all its neighbors. Then all the neighbor nodes forward it to their nearest neighbors and so on. After this, nodes send RREP packets to their neighbors from which they first receive the RREQ. These RREP packets which are transmitted to the intermediate nodes update the routing tables. These table entries indicate the node from which the reply packets (RREP) were received. Due to the dynamic nature of the nodes in ad-hoc network, when the links between nodes are broken, it's necessary to start a route repair function. If any node is not in the transmission range of the neighbor node and if links are broken between the nodes, then neighbor node (upstream neighbor) broadcasts a Routing Error (RERR) packet which is a link broken information or notification to each of its neighbor. After this a new route discovery process is starts by the source node. Because of VANET unique characteristics like highly dynamic nature, if we directly apply traditional AODV protocol it gives poor performance. In order to get good results of AODV protocol in a realistic VANET scenario, there are lots of works have been done to enhance AODV. In the next section we present a number of AODV enhancements which are proposed by different authors yearly in order to improve AODV performance and make it applicable in VANET.
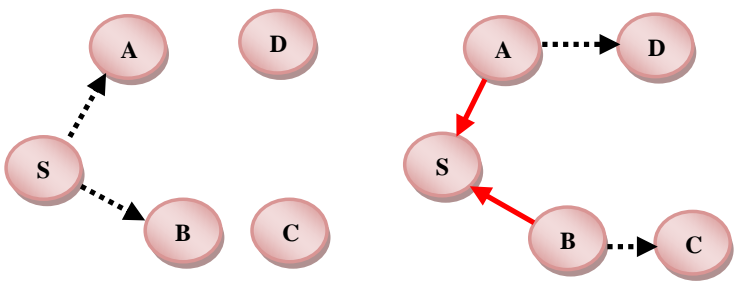

i. S wants to send a packet to $D$ S broadcasts RREQ packets

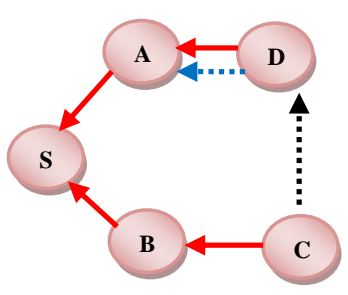
ii. A \& B establish Reverse Route A \& B rebroadcast RREQ packet

\section{iii. C \& D establish Reverse Route C rebroadcasts RREQ D unicasts RREP}

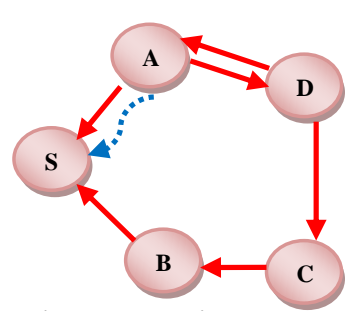

iv. D establishes

Reverse Route $D$ drops duplicate RREQ

A establishes Route A reunicasts RREP

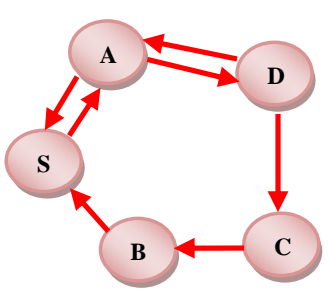

v. S establishes Route

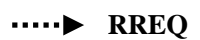

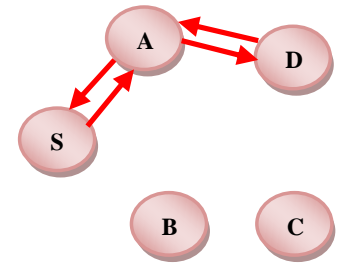

vi. Unused Reverse Route expire
ROUTE

Figure 4: Route Establishment Procedure in AODV

\section{SEVERAL AODV ENHANCEMENTS IN VANETs}

There are many AODV enhancements which are proposed by different authors.

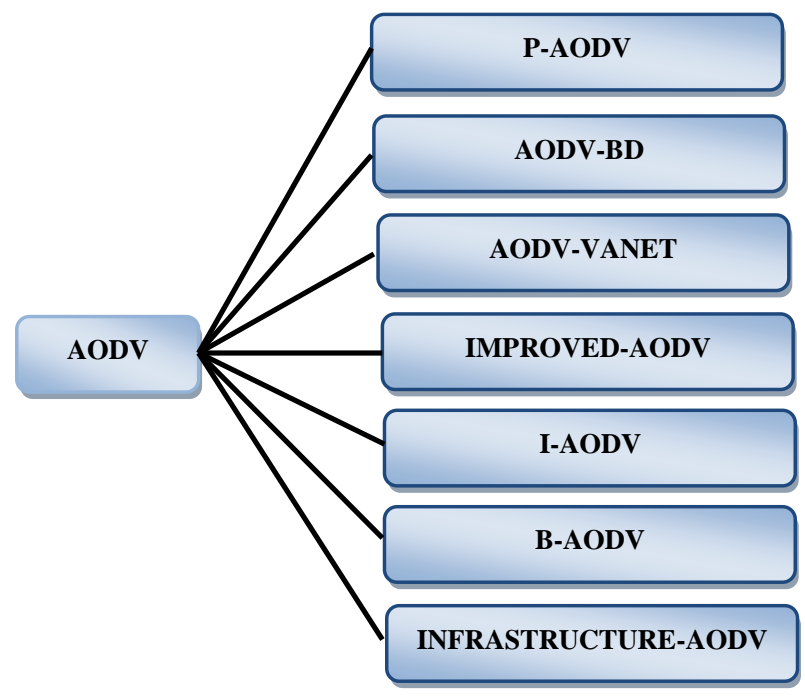

Figure 5: Several AODV enhancements in a realistic VANET context 
All have taken different approaches and parameters to design a suitable protocol which gives better performance in VANET environment. Figure 5 illustrates some of the enhanced protocols of AODV that are work in VANETs.

\subsection{P-AODV}

To reduce control overhead over the network Omid Abedi et al. [12] improved AODV protocol and designed PriorAODV. In this protocol, nodes are restricted by which they can't get the RREQ packets and by doing this number of discovered routes are decreased and as a result network overhead is reduced. In P-AODV, neighbors of any node divided into two categories based on their distance from source node; first category is prior neighbor and the second category is overhead neighbor. Both neighbors are present in their zone i.e. prior neighbor in prior zone and overhead neighbor in overhead zone. Prior zone of any node is the distance between threshold zone and transmission range of that node and overhead zone lies between node \& respective threshold distance. In this protocol, broadcasting of RREQ packets is determined using two parameters.

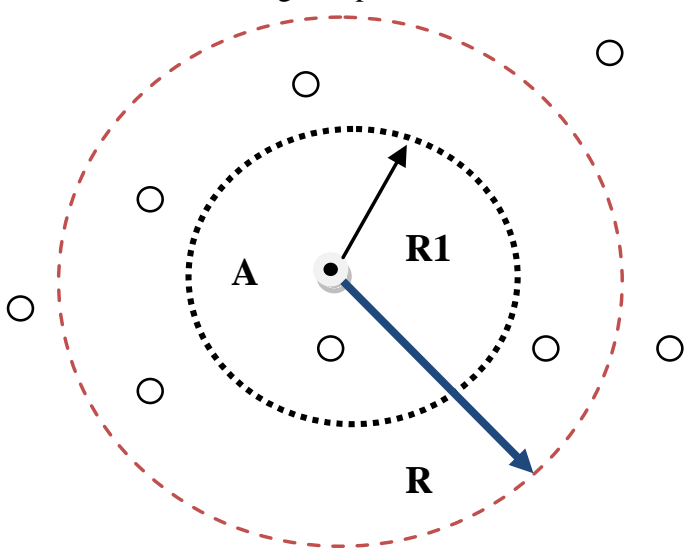

\section{R: Transmission range of node $A$ R1: Threshold distance}

\section{Figure 6: Prior \& Overhead neighbor concept used in P-} AODV

In first parameter, RREQ packets are restricted by which routes are limited. In second parameter, nodes are selected for which these restricted RREQ packets be sent to them. For the route discovery process, nodes first have to find their neighbor's type either it is overhead neighbor or it is prior neighbor. To implement this P-AODV protocol author uses GloMoSim library. The result shows that this protocol reduces the overhead and length of the route for data transmission in VANET environment.

\subsection{AODV-BD}

To reduce the packet delay in the network Baozhu $\mathrm{Li}$ et al. [13] enhance AODV protocol and make a protocol called AODV-BD. The concept behind in this protocol is that data packets are broadcasted even when the broken links are repaired. When there is a broken link, rather than sending a RREQ, node directly broadcasts a packet by which packet delay gets reduced and then the destination node transmits a RREP packet after receiving the data packets. The mobility model which is used to implement in this protocol is VANETMobiSim and the simulation is performed using NS2 .

\subsection{AODV-VANET}

Huaqun Guo et al. [14] enhance the common AODV protocol which we used in MANET and develop a protocol called AODV-VANET. The key consideration behind this protocol is to achieve an optimal path from a source to a destination for better delivery of data packets. To design AODV-VANET protocol, in the route discovery process weight of the route from source to destination node is calculated and added to the original AODV protocol. This optimized AODV-VANET protocol modifies RREQ \& RREP messages in the route discovery phase. In RREQ message, source node initially added some information in RREQ like node speed, acceleration detail etc... In RREP, more weight and routing path is added in it and packet is sent to the source node. By this, the source node selects the best possible path with minimum amount of route weight on it among many possible routes. To implement AODV-VANET author used TraNS to generate realistic scenario and simulation is performed using NS-2.

\subsection{Improved-AODV}

Zehua Chen et al. [15] proposed an enhance form of AODV called Improved-AODV. The main benefits of this enhancement are that this protocol provides stability between routes i.e. from source to destination and also reduces network overhead. To achieve this author performed two steps of optimization procedures in route discovery \& route selection phases. For the routing stability, it is important that the vehicles move in the opposite direction or in the same direction because if the vehicles are in the same direction then they will be in communication range for a longer time period as compare to opposite direction vehicles. Following are the two steps which are proposed by author to optimize the AODV protocol.

In first step, in route discovery phase, only those nodes are selected which have more stable links than others. In this procedure RREQ packets are broadcasted to only those vehicles which have the similar speed to the source vehicle and the direction is also same as the source vehicle direction. By this, the overhead is reduced because RREQ is not broadcasted to the whole network. In the second step, when there are multiple routes to source to destination then source node selects a route which is more stable. Route selection procedure is carried out by two different strategies; in first strategy, the route is selected by calculating the Route Expiration Time (RET). Because longer route is more reliable for the transmission of data packets so this strategy is used. The calculation of RET is performed using the Link Expiration Time (LET), which route have minimum LET that route is selected for transmission. In second strategy, selection of route is performed using total weight. The routes which have minimum weight are chosen. By using this strategy, the stability of a route can be easily determined. The performance comparison is done using different parameter like speed of nodes, number of vehicles.

\subsection{I-AODV}

Dharmendra Sutariya et al. [16] propose a protocol called IAODV (Improved). The main goal of this protocol is to provide accurate information \& timely delivery of data packets. Author defines I-AODV protocol as "Limited Source Routing up To Two Hops with Backup Route Between Source Node and Destination Node." This hybrid protocol combines mechanism of DSR and AOMDV into the AODV protocol and make I-AODV. This protocol works on two phases; Route Discovery \& Route Maintenance. In first phase, route request 
is getting modified for limited source routing. This limited source routing is performed only up to two hops. In route reply there is a backup route mechanism between source node and destination node. In second phase, if there is a link broken or primary route is failed then source node transmitted data by backup route. If backup route is also failed then the new route discovery procedure is begin by source node. To implement IAODV the authors take a realistic scenario using MOVE and simulation is performed using NS-2.

\subsection{B-AODV}

In order to reduce end-to-end delivery of data packets in a VANET, Sufyan T. Faraj Al-Janabi et al. [17] proposed an enhance AODV called Bus-AODV. The key idea behind this protocol is that it only works for either a car or a bus. In the proposed model, the protocol selects only those routes in which there is less number of buses as compare to other vehicles. The advantage of this technique is that it reduces end-to-end delay in the network. The two important conditions for which author performs changes in AODV.CC file is; sequence number and hop count. Rather than select lower hop count for communication the proposed protocol chooses vehicle type i.e. it selects buses for packet forwarding.

In this paper the proposed protocol B-AODV is designed mainly for sensing buses. The new parameters which are added in order to modify RREP \& RREQ are; number of vehicles and the number of buses through route. The author used NS-2 to perform the simulation of Bus-AODV. The mobility model which is used is Downtown. The performance of this protocol is analyzed by using various performance metrics such as Normalized Routing Load (NRL), End-to-End (E2E) delay, Packet Delivery Ration (PDR). These analyses show that the performance of B-AODV is far better than the traditional AODV in Downtown and similar mobility model. The comparative analysis of B-AODV and other enhance AODV protocol is shows in the next section.

\subsection{Infrastructure-AODV}

Faisal Iqbal et al. [18] proposed an enhance form of AODV protocol called Infrastructure-AODV. In this protocol, fixed infrastructure i.e. RSUs are used to provide path information to the vehicles and directional antennas are used to gain the Line of Sight (LOC) communication among them. In this proposed protocol communication is achieved by fixed nodes so it's more reliable and fast. This protocol broadcast HELLO messages to find free routes and existing routes are validated.

\section{RELATIVE COMPARISON OF} SEVERAL AODV ENHANCEMENTS

After studying various AODV enhancements, we analyze performance of all the protocols. Table 2 gives a relative comparison of all the above discuss enhanced AODV protocols:

\section{CONCLUSION \& FUTURE WORK}

In this paper, we have studied and analyzed various AODV enhancements in context to realistic VANET. We have also done the comparative study of all the existing enhanced AODV routing protocols using different performance metrics and then found that the enhanced AODV protocols performed inadequate for some of the performance metrics.

Our future work will mainly focus on to design a robust AODV protocol for VANET which can provide a better performance as compare to other relative protocols.

\begin{tabular}{|c|c|c|c|c|c|c|c|c|c|c|}
\hline PROTOCOLS & $\begin{array}{c}\text { Simulation } \\
\text { Time } \\
\text { (sec.) }\end{array}$ & $\begin{array}{l}\text { Directional } \\
\text { - Antenna }\end{array}$ & $\begin{array}{l}\text { Propagation } \\
\text { Model }\end{array}$ & $\begin{array}{c}\text { Node } \\
\text { Density }\end{array}$ & Velocity & PDR & NRL & $\begin{array}{l}\text { Packet } \\
\text { Delay }\end{array}$ & $\begin{array}{l}\text { Broken } \\
\text { Links }\end{array}$ & $\begin{array}{c}\text { Traffic } \\
\text { Type }\end{array}$ \\
\hline P-AODV & 1000 & Uniform & $\begin{array}{l}\text { Random } \\
\text { waypoint }\end{array}$ & Medium & Medium & ND & ND & ND & Low & ND \\
\hline AODV-BD & 300 & Omni & $\begin{array}{l}\text { Two Ray } \\
\text { Ground }\end{array}$ & Low & Low & High & ND & Low & ND & $\begin{array}{l}\text { TCP \& } \\
\text { CBR }\end{array}$ \\
\hline AODV-VANET & 1800 & Omni & ND & High & High & ND & High & ND & ND & TCP \\
\hline IMPROVED-AODV & ND & Uniform & $\mathrm{ND}$ & Medium & Low & High & ND & ND & High & ND \\
\hline I-AODV & 400 & Omni & $\begin{array}{l}\text { Two Ray } \\
\text { Ground }\end{array}$ & Medium & Low & High & Low & Medium & ND & TCP \\
\hline B-AODV & 300 & Omni & $\begin{array}{l}\text { Two Ray } \\
\text { Ground }\end{array}$ & Low & Medium & Medium & Medium & Low & ND & CBR \\
\hline $\begin{array}{l}\text { INFRASTRUCTURE- } \\
\text { AODV }\end{array}$ & 360 & Omni & ND & Medium & Medium & ND & ND & Medium & ND & CBR \\
\hline
\end{tabular}




\section{REFERENCES}

[1] Naumov, V., R. Baumann, and T. Gross. An evaluation of inter-vehicle ad hoc networks based on realistic vehicular traces. in Proceedings of the 7th ACM international symposium on Mobile ad hoc networking and computing. 2006: ACM.

[2] Azogu, I.K., M.T. Ferreira, and L. Hong. A security metric for VANET content delivery. in Global Communications Conference (GLOBECOM), 2012 IEEE. 2012.

[3] Olariu, S. and M.C. Weigle, Vehicular networks: from theory to practice. 2010: CRC Press.

[4] Uzcategui, R. and G. Acosta-Marum, Wave: A tutorial. Communications Magazine, IEEE, 2009. 47(5): p. 126133.

[5] Jiang, D. and L. Delgrossi. IEEE 802.11p: Towards an International Standard for Wireless Access in Vehicular Environments. in Vehicular Technology Conference, 2008. VTC Spring 2008. IEEE. 2008.

[6] Jerbi, M., et al., Vehicular Communications Networks: Current Trends and Challenges. IGI Global, 2010: p. 251-262.

[7] Dua, A., N. Kumar, and S. Bawa, A systematic review on routing protocols for Vehicular Ad Hoc Networks. Elsevier Inc., 2014(Vehicular Communications1(2014)33-52): p. 20.

[8] Taha, M.M.I., Broadcasting protocols in vehicular ad-hoc networks (vanets). MSc., Electrical Engineering, Assuit University, 2008.

[9] Boukerche, A., et al., Vehicular ad hoc networks: A new challenge for localization-based systems. Computer communications, 2008. 31(12): p. 2838-2849.

[10] Ledy, J., et al. AODV enhancements in a realistic VANET context. in Wireless Communications in Unusual and Confined Areas (ICWCUCA), 2012 International Conference on. 2012.
[11] Perkins, C.E. and E.M. Royer. Ad-hoc on-demand distance vector routing. in Mobile Computing Systems and Applications, 1999. Proceedings. WMCSA'99. Second IEEE Workshop on. 1999: IEEE.

[12] Abedi, O., R. Berangi, and M.A. Azgomi. Improving route stability and overhead on AODV routing protocol and make it usable for VANET. in Distributed Computing Systems Workshops, 2009. ICDCS Workshops' 09. 29th IEEE International Conference on. 2009: IEEE.

[13] Li, B., Y. Liu, and G. Chu. Improved AODV routing protocol for vehicular Ad hoc networks. in Advanced Computer Theory and Engineering (ICACTE), 2010 3rd International Conference on. 2010: IEEE.

[14] Guo, H., et al. An optimized routing protocol for vehicular ad hoc networks. in TENCON 2010-2010 IEEE Region 10 Conference. 2010: IEEE.

[15] Ding, B., et al. An improved AODV routing protocol for VANETs. in Wireless Communications and Signal Processing (WCSP), 2011 International Conference on. 2011: IEEE.

[16] Sutariya, D. and S. Pradhan. An improved AODV routing protocol for VANETs in city scenarios. in Advances in Engineering, Science and Management (ICAESM), 2012 International Conference on. 2012: IEEE.

[17] Al-Janabi, S.T.F., Y.S. Yaseen, and B. Askwith, The Bus Ad Hoc On-demand Distance Vector (BAODV) Routing Protocol. 2012: PGNet.

[18] Iqbal, F., et al. I-AODV: Infrastructure based Ad Hoc On-demand Distance Vector routing protocol for Vehicular Ad Hoc Networks. in Smart Instrumentation, Measurement and Applications (ICSIMA), 2013 IEEE International Conference on. 2013: IEEE. 